\title{
UCRL-TR-212809
}

LAW RENCE LIVERMORE N A T IO N A L LABORATORY

\section{The LCLS Gas Attenuator Revisited}

D.D. Ryutov

June 9, 2005 
This document was prepared as an account of work sponsored by an agency of the United States Government. Neither the United States Government nor the University of California nor any of their employees, makes any warranty, express or implied, or assumes any legal liability or responsibility for the accuracy, completeness, or usefulness of any information, apparatus, product, or process disclosed, or represents that its use would not infringe privately owned rights. Reference herein to any specific commercial product, process, or service by trade name, trademark, manufacturer, or otherwise, does not necessarily constitute or imply its endorsement, recommendation, or favoring by the United States Government or the University of California. The views and opinions of authors expressed herein do not necessarily state or reflect those of the United States Government or the University of California, and shall not be used for advertising or product endorsement purposes.

This work was performed under the auspices of the U.S. Department of Energy by University of California, Lawrence Livermore National Laboratory under Contract W-7405-Eng-48. 


\title{
LCLS Technical Memo.
}

\section{The LCLS Gas Attenuator Revisited}

\author{
D.D. Ryutov
}

In the report "X-ray attenuation cell" [1] a preliminary analysis of the gas attenuator for the Linac Coherent Light Source (LCLS) was presented. This analysis was carried out for extremely stringent set of specifications. In particular, a very large diameter for the unobstructed beam was set $(1 \mathrm{~cm})$ to accommodate the spontaneous radiation; the attenuator was supposed to cover the whole range of energies of the coherent radiation, from $800 \mathrm{eV}$ to $8000 \mathrm{eV}$; the maximum attenuation was set at the level of $10^{4}$; the use of solid attenuators was not allowed, as well as the use of rotating shutters.

The need to reach a sufficient absorption at the high-energy end of the spectrum predetermined the choice of $\mathrm{Xe}$ as the working gas (in order to have a reasonable absorption at a not-too-high pressure). A sophisticated differential pumping system that included a Penning-type ion pump was suggested in order to minimize the gas leak into the undulator/accelerator part of the facility. A high cost of xenon meant also that an efficient (and expensive) gas-recovery system would have to be installed.

The main parameter that determined the high cost and the complexity of the system was a large radius of the orifice. The present viewpoint allows for much smaller size of the orifice,

$$
r_{0}=1.5 \mathrm{~mm} \text {. }
$$

The use of solid attenuators is also allowed (R.M. Bionta, private communication). It is, therefore, worthwhile to reconsider various parameters of the gas attenuator for these much less stringent conditions. This brief study should be considered as a physics input for the engineering design.

As a working gas we consider now the argon, which, on the one hand, provides a reasonable absorption lengths and, on the other hand, is inexpensive enough to be exhausted into the atmosphere (no recovery). The absorption properties of argon are 
illustrated by Fig. 1 where the attenuation factor $A$ is shown for various beam energies, based on Ref. [2]. The other relevant parameters for argon are presented in Table 1.
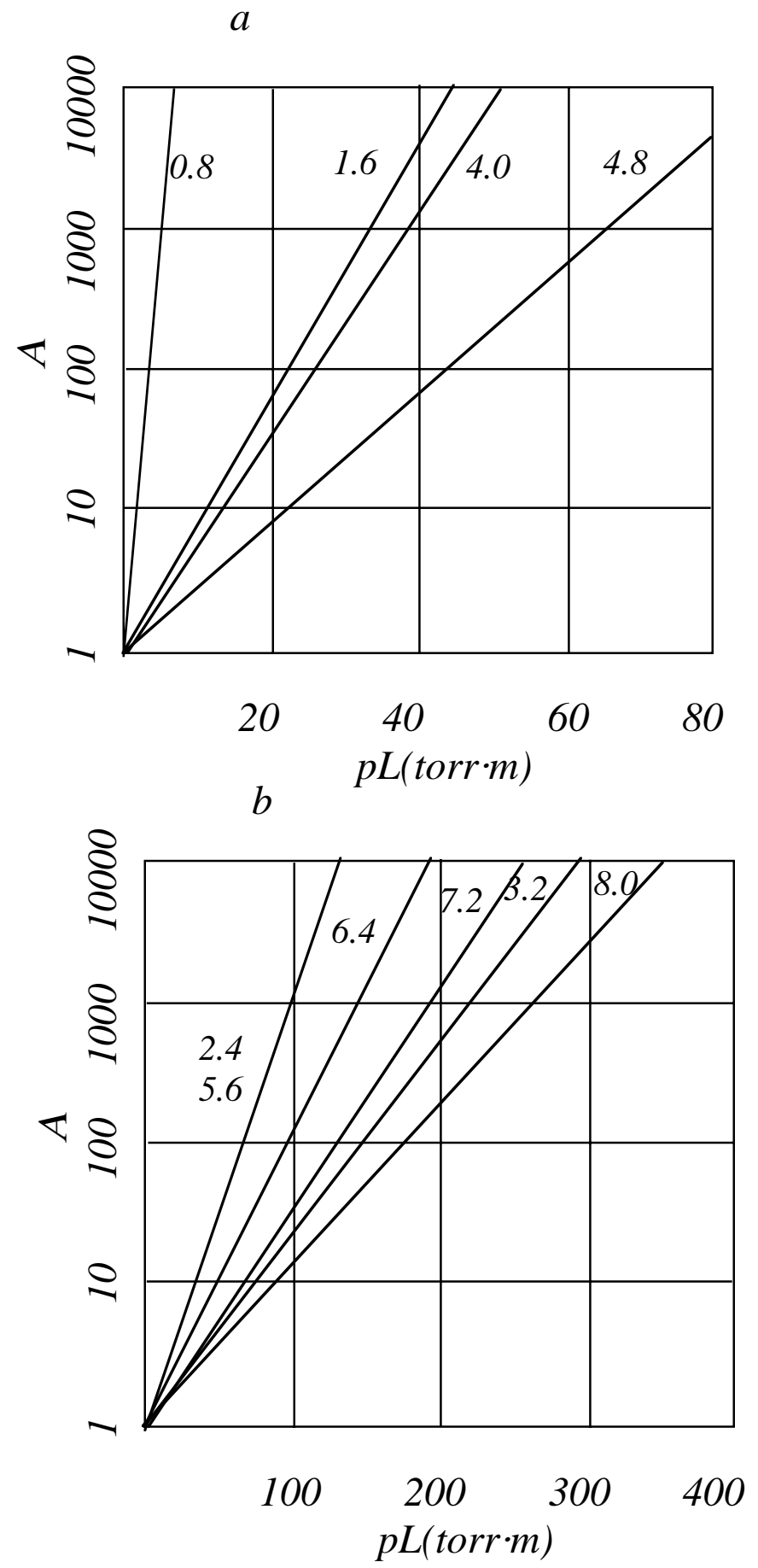

Fig.1 The dependence of the attenuation coefficient $(A)$ on the line-density of argon in the attenuation cell. The numbers by the lines indicate the X-ray energy in $\mathrm{keV}$. We split the figure into two panels because of the difference of the horizontal scales. The lines in panel (b) for the energies 2.4 and $5.6 \mathrm{keV}$ are virtually indistinguishable at this scale. 
Table 1. Some parameters of argon

\begin{tabular}{|c|c|c|c|c|c|}
\hline $\begin{array}{c}\text { Atomic } \\
\text { number }\end{array}$ & $\begin{array}{c}\text { Atomic } \\
\text { radius [3] }\end{array}$ & $\begin{array}{c}\text { Collision } \\
\text { cross- section }\end{array}$ & $\begin{array}{c}\text { Density at } \\
\text { normal } \\
\text { cond. }\end{array}$ & $\begin{array}{c}\text { Sound speed } \\
\text { at normal } \\
\text { cond. }\end{array}$ & $\begin{array}{c}\text { Viscosity, } \\
\text { at } 0^{0} \mathrm{C}[4]\end{array}$ \\
\hline $\mathrm{A}=40$ & $r_{a}=98 \AA$ & $\begin{array}{c}\sigma=1.21 \cdot 10^{-15} \\
\mathrm{~cm}^{2}\end{array}$ & $\begin{array}{c}\rho_{0}=1.78 \cdot \\
10^{-3} \mathrm{~g} / \mathrm{cm}^{3}\end{array}$ & $s_{0}=320 \mathrm{~m} / \mathrm{s}$ & $\begin{array}{c}\eta=2.1 \cdot 10^{-4} \\
\mathrm{~g} / \mathrm{s} \cdot \mathrm{cm}\end{array}$ \\
\hline
\end{tabular}

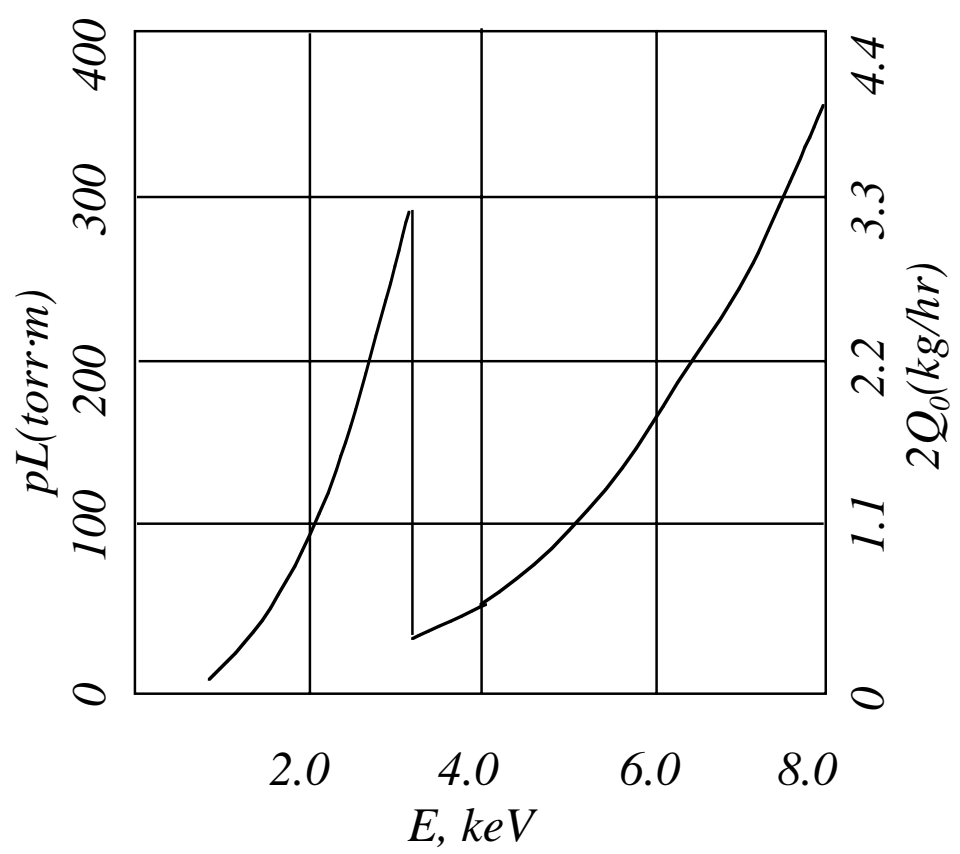

Fig. 2 The argon line density required to produce a $10^{4}$ attenuation at various photon energies (left scale), and the un-inhibited exhaust from two 3-mm diameter orifices from the attenuation cell, for the length of the attenuation cell $L=2 \mathrm{~m}$ (right scale). For the attenuation $A=100$ the vertical scale shoulfd be divided by a factor of 2 ; for the attenuation $A=10$, the vertical scale should be divided by a factor of 4 .

Figure 2 shows the line density of argon required to reach an attenuation of $10^{4}$. Assuming that the length of the main attenuation cell is $L=2 \mathrm{~m}$, one sees that, to reach the highest attenuation of $A=10^{4}$, one has to have the pressures in this cell in the range between $\sim 5$ torr and $\sim 175$ torr. The mean free path for the argon atoms, evaluated as

$$
\lambda=\frac{1}{n \sigma}
$$

(where $n$ is the particle density, and $\sigma$ is the collision cross-section, see Table 1) in this range of pressures lies between a few hundred microns and a fraction of a micron (Fig. 
3). This means that the gas flow is collisional in the sense that the mean free path is much shorter than the orifice radius $r_{0}$. This, in turn, means that the gas exhaust through each of the orifices will occur in a hydrodynamic manner (not in a Knudsen manner); the discharge through each of the orifices can then be evaluated by Eq. (14) of Ref. [1]:

$$
Q_{0}=\pi r_{0}^{2} s_{0} n_{0}\left(\frac{2}{\gamma+1}\right)^{\frac{\gamma+1}{2(\gamma-1)}},
$$

where the quantities bearing a subscript " 0 " refer to the attenuation cell, and $\gamma=5 / 3$ is the adiabatic index for argon. Taking $r_{0}$ from Eq, (1) and assuming that the argon in the cell is at a room temperature, one can rewrite this expression as:

$$
Q_{0}(\text { atoms } / s)=4.6 \cdot 10^{19} p_{0}(\text { torr }),
$$

where $p_{0}$ is the pressure in the central absorbing cell. Alternatively, this can be presented as:

$$
Q_{0}(\mathrm{~g} / \text { hour })=11 p_{0}(\text { torr }) \text {. }
$$

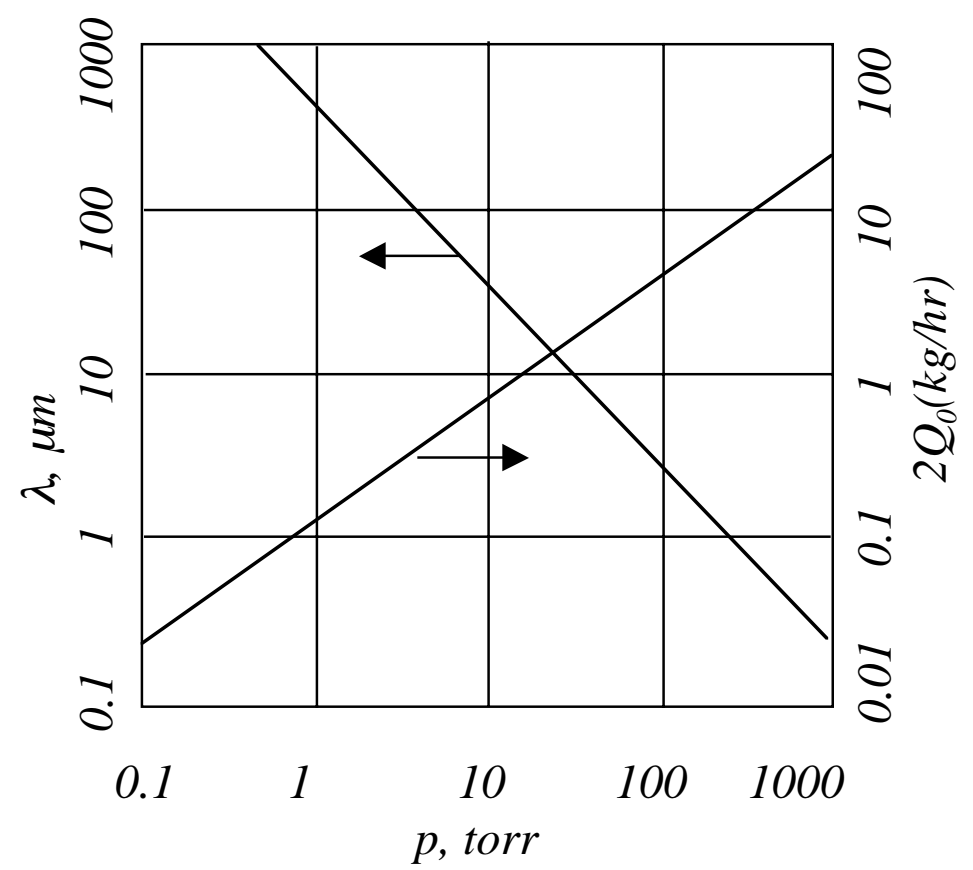

Fig. 3 The mean-free-path (left scale) and the un-inhibited argon out flow from two ends (right scale) vs the argon pressure.

The right scale in Fig. 2 shows the gas consumption for $L=2 \mathrm{~m}$ and the attenuation $A=10^{4}$. Except for the lowest energies $<1 \mathrm{keV}$, the consumption is in the range of kilograms/hour. This is high, both from the viewpoint of the cost of the gas (see below), 
the demands to the differential pumping system, and the leaks to the accelerator volume. So, the gas flow has to be reduced by a factor of 10 or more.

This can be done by attaching a tube with multiple diaphragms to the exhaust orifice (Fig.4; Cf. also Fig.3 in Ref. [1]). The orifices in the diaphragms would have the same diameter as the exit orifice (i.e., $3 \mathrm{~mm}$ in our main example), The tube radius can be made larger than the diameter of a spontaneous halo, not to affect its propagation (if this halo is needed for the experiments or calibrations). The total thickness of all diaphragms would be small enough not to affect this spontaneous halo.

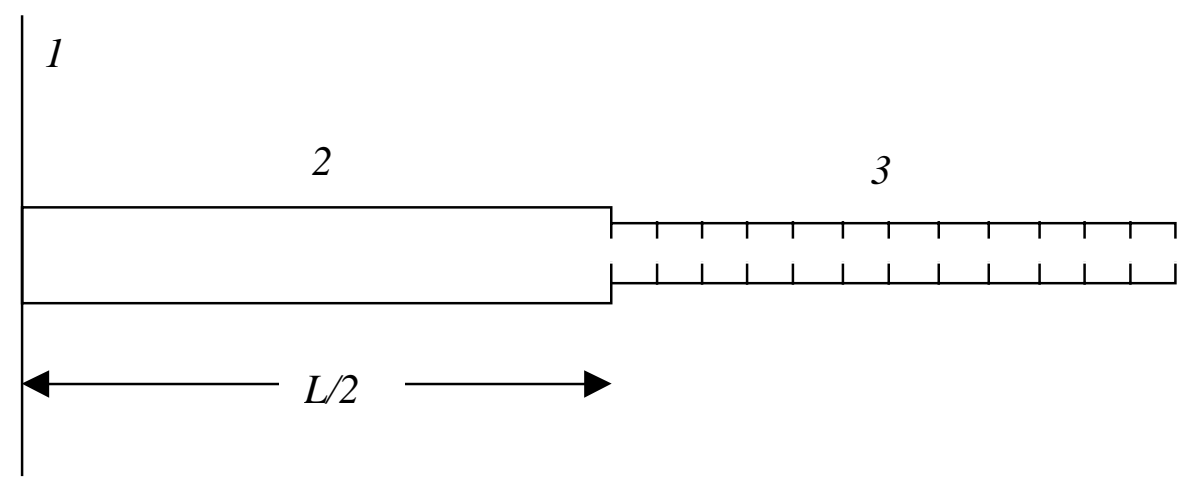

Fig. 4 A rough schematic of the attenuator (geometrical dimensions are not to scale): 1 a symmetry plane; 2 - central attenuation cell; 3 - a high gas-resistance tube.

We will characterize the reduction of the gas loss by this high-gas-resistance (HGR) tube by a coefficient $K$ which will be 10 at the highest pressure; it will increase substantially towards the lower pressures). The gas discharge from each end of the central section will be

$$
Q=Q_{\delta} / K,
$$

and the total discharge from two ends will be, obviously, by a factor of 2 higher.

As a numerical example, consider the pressure of 176 torr, which corresponds to the attenuation of $10^{4}$ for $8-\mathrm{keV}$ beam in a 2-m long central cell. The total discharge in grams/hour, is, according to Eqs. (5-6): $2 \times 11 \times 176 / 10=387$. [This corresponds to 8 cubic feet at normal conditions.] Note that this amount corresponds to the most demanding mode of operation, at the highest energy and at the highest attenuation coefficient. During 1 year of a continuous operation, the consumption of argon would therefore not exceed 
3.3 t. The cost of this argon will be in the range of a few tens of thousands dollars (depending on its purity). For the operation at low energy $(0.8 \mathrm{keV})$ the amount of gas will be smaller by at least an order of magnitude. So, we conclude that there is no need in the gas recovery system.

To compare argon with another possible absorbing gas, the nitrogen, we present Fig. 5. One sees that argon is a significantly better absorber, especially at the higher end of the spectrum.

The differential pumping system beyond the exit of the tube 1 will be a standard differential pumping system. Its specific design would depend on the allowed amount of gas leak to the undulator. The amount of gas that will have to be pumped out from the section that follows the end of the tube 1 will vary by at least two orders of magnitude between $8-\mathrm{keV}$ and $0.8 \mathrm{keV}$ beam energy.

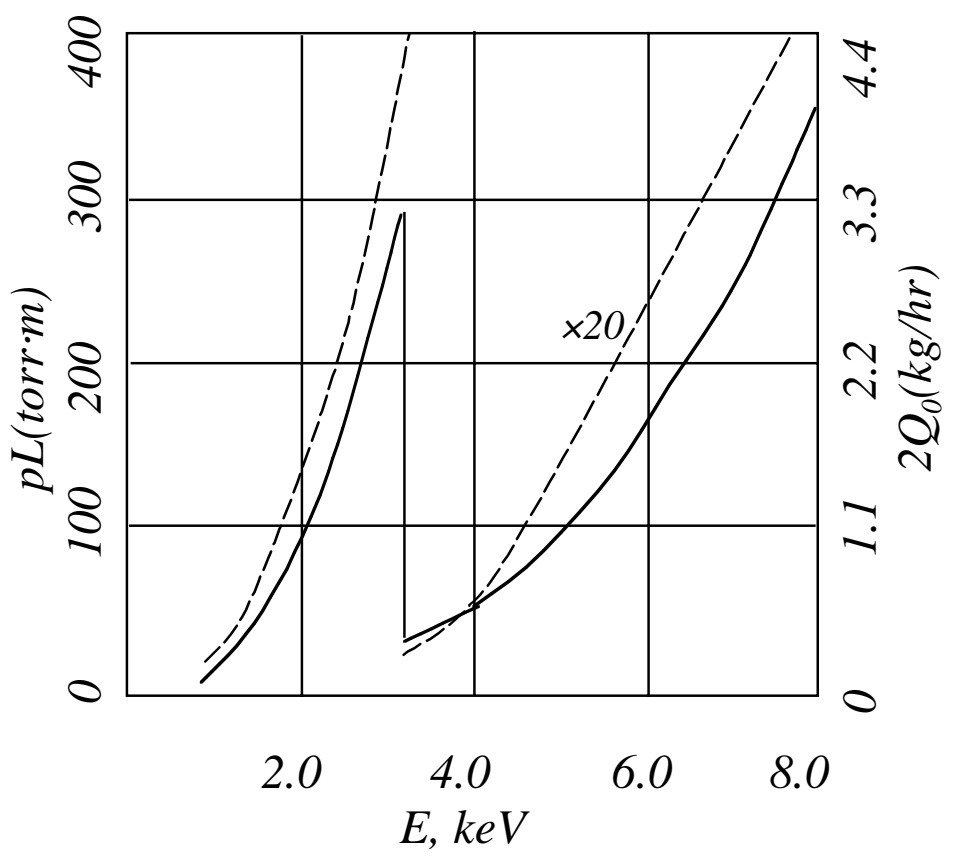

Fig. 5. Comparison of the absorption properties of argon and nitrogen. Shown is the line density required for reaching an attenuation of $10^{4}$ for argon (solid line, the same as in Fig. 2) and for nitrogen (dashed line). At the energies exceeding $3.2 \mathrm{keV}$, the scale for nitrogen has to be multiplied by 20 (e.g., at $8 \mathrm{keV}$, the line density for nitrogen is $\sim 8500$ torr'm). 
Another challenging issue is the accuracy to which the attenuation coefficient can be maintained. The relative variation of the attenuation coefficient can be expressed in terms of a relative variation of the line density as:

$$
\frac{\Delta A}{A}=\frac{\Delta(p L)}{p L} \ln A
$$

So, to have a $1 \%$ accuracy at an attenuation of $A=100$, one would have to maintain the gas pressure with the accuracy of order of $0.1 \%$. This seems to be a very difficult task. A question of what is the level of $\triangle A / A$ required by the experimentalists and how it is related to the accuracy of maintaining the pressure has to be answered as soon as possible. One more problem is associated with the fact that some gas will be present not only in the central attenuation cell but also in the HGR tubes. It will contribute to the line density. This will be especially important at the highest attenuations $\sim 10^{4}$. In order to know what is the attenuation coefficient at a given central-cell pressure, one would have to calibrate the system at various pressures (or gas throughputs) and various X-ray energies. This can be done before installing the attenuator at the LCLS. Periodic calibrations can, in principle, be done in the low-intensity LCLS mode.

At the X-ray energies in the range below $1.5 \mathrm{keV}$ one can use a very simple attenuator, without the HGR tube attached, especially if the required attenuation is below 100. In this case, the gas loss will be below $~ 300 \mathrm{~g} / \mathrm{hr}$. So, one can think of different types of the attenuators for the lowest energies and for the rest of the spectrum. Of course, the need to switch from one to another between experimental campaigns adds to cost and complexity.

To facilitate the further work on the gas attenuator, we have to clarify the following issues:

1. What is the main function of the gas attenuator (is it predominantly a simple and reliable beam dump, or is it an experimental tool that will be widely used by the experimental groups)?

2. Where the need to have a $10^{4}$ attenuation comes from? Wouldn't it be sufficient to have a maximum attenuation of 100 ? 
3. What dictates the need of $1 \%$ accuracy at the highest attenuation? Reaching such an accuracy is difficult, especially at the higher X-ray energies. More generally, what the users would want from the gas attenuator?

4. How much material can be placed at the radii between $1.5 \mathrm{~mm}$ and $10 \mathrm{~mm}$ ? - This relates to an issue of the effect on a high-energy halo.

5. What is the allowed gas leak from the gas attenuator to the undulator?

\section{Acknowledgment.}

I am grateful to Dr. R.M. Bionta for his support of this study, and to R. Wood for providing an information about the cost of argon. This work was performed under the auspices of the U.S. Department of Energy by University of California Lawrence Livermore National Laboratory under Contract W-7405-Eng-48. 


\section{References.}

1. D.D. Ryutov, A. Toor. "X-ray attenuation cell.” UCRL-ID-138125, LCLS Technical Note 00-10, May 2000.

2. B.L. Henke, E.M. Gullikson, J.C. Davis. Atomic Data and Nuclear Data Tables, 54, 181 (1993).

3. http://www.scescape.net/ woods/elements/argon.html

4. “'A Physicist's Desk Reference,” H.L. Anderson, Ed., AIP, NY, 1989. 